POPULATION: We conducted interviews with 107 stakeholders including patients who have had strokes, rehab directors, and physical/occupational therapists to understand their viewpoints for adopting new rehabilitation devices. To contribute to previous literature, interviews were analyzed qualitatively using direct content analysis to provide more specific details about the most appropriate adoption settings, specific roles for stakeholders, and drivers for all stakeholders involved in the adoption process. RESULTS/ANTICIPATED RESULTS: Unique to this work, care settings in which therapy goals are best aligned for restorative devices were found to be outpatient rehabilitation, followed by inpatient rehabilitation. Therapists are the major influencers for adoption because they typically introduce new rehabilitation devices to patients for both clinic and home use. We also learned therapists' utilization rate of a rehabilitation device influences a rehabilitation director's decision to acquire the device for facility use. Additionally, device setup in $<7$ minutes will allow for increased use without reducing therapist productivity. DISCUSSION/SIGNIFICANCE OF FINDINGS: Rehabilitation device development should consider the best settings to first introduce the device, roles of each stakeholder, and drivers that influence each stakeholder to accelerate successful adoption of the developed device.

69399

How are substance use disorder treatment programs in Arkansas responding to COVID-19? A qualitative study

Jure Baloh ${ }^{1}$ and Geoffrey M. Curran ${ }^{2}$

${ }^{1}$ University of Arkansas for Medical Sciences and ${ }^{2}$ University of

Arkansas for Medical Sciences, Central Arkansas Veterans

Healthcare System

ABSTRACT IMPACT: This study informs how substance use treatment programs responded to the COVID-19 pandemic, and highlights implication for future translational research and practice. OBJECTIVES/GOALS: The COVID-19 pandemic rapidly changed how substance use disorder (SUD) treatment services are organized and provided. This study examined what changes SUD treatment programs in Arkansas implemented (e.g., guidelines, technologies), and what factors influenced their ability to implement and sustain these changes. METHODS/STUDY POPULATION: Between May and August 2020, we conducted semi-structured phone interviews with 29 leaders (administrative and/or clinical leaders) at 21 residential and outpatient SUD treatment programs throughout Arkansas (i.e., in all five Arkansas public health regions). Interviews were based on the Consolidated Framework for Implementation Research and focused on what changes programs were implementing in response to the COVID-19 pandemic, barriers and facilitators to implementation, and recommendations for future. The interviews were on average about 30 minutes long, and we provided no participant compensation. Interviews were recorded and transcribed verbatim, then thematically analyzed. RESULTS/ANTICIPATED RESULTS: Programs implemented similar infection control practices: screening at entry, masks, hand hygiene, and social distancing. Residential programs stopped outside visitations and some capped admissions; outpatient programs stopped group sessions and switched most services to telehealth. Key facilitators included grants/loans (e.g., salaries), looser regulatory restrictions (e.g., telehealth), and good coordination with other organizations (e.g., state agencies). Key barriers included limited access to supplies (e.g., masks), no rapid testing (particularly for residential care), limited capacity for social distancing, and negative employee and client responses (e.g., anxiety). Key recommendations include better access to supplies and testing, telehealth continuation and better communication. DISCUSSION/ SIGNIFICANCE OF FINDINGS: This study provides an insight into how SUD programs responded to the COVID-19 pandemic and what the 'new normal' is. This can inform D\&I studies conducted in SUD settings, including studies examining what implementation strategies can help sustain these changes, or studies of other practices implemented during or after the pandemic.

84539

\section{Developing a Multilevel Intervention to Increase Hepatitis C Virus Screening of Baby Boomers in Primary Care \\ Monica Kasting ${ }^{1}$ and Susan Rawl ${ }^{2}$ \\ ${ }^{1}$ Purdue University and ${ }^{2}$ Indiana University School of Nursing}

ABSTRACT IMPACT: This research will improve human health by increasing screening for hepatitis $\mathrm{C}$ virus, thereby decreasing morbidity and mortality from hepatitis C-related disease. OBJECTIVES/GOALS: The worldwide incidence of liver cancer increased 75\% from 1990 to 2015 due, in part, to chronic hepatitis C virus (HCV) infection. Individuals born 1945-1965 (baby boomers) have five times the prevalence of HCV infection compared to other birth cohorts, but fewer than $15 \%$ of this cohort have ever been screened. METHODS/STUDY POPULATION: Effective interventions to increase HCV screening among baby boomers are urgently needed. In partnership with a provider advisory board and a community advisory board, we will develop a multilevel intervention designed to increase HCV screening that will be delivered to both providers and patients in primary care. We will assess whether the intervention is feasible, acceptable, and usable from the perspectives of the target audiences (providers and patients) by conducting Concurrent Think Aloud (CTA) interviews with eight patients and eight providers. RESULTS/ANTICIPATED RESULTS: While the specific content of both intervention components will not be finalized until the completion of the study, we envision that the provider-level intervention will likely include a one-time educational session and monthly performance feedback provided via e-mail reporting each provider's HCV screening rates. The patient-level intervention may include mailed reminder letters prior to a scheduled clinic visit informing them that $\mathrm{HCV}$ screening is recommended and a tablet-based in-clinic computer program to educate, engage, and activate patients to be screened. DISCUSSION/SIGNIFICANCE OF FINDINGS: The goals of this project are to: 1) develop an acceptable, feasible, and usable multilevel intervention aimed at increasing HCV screening in primary care; and 2) understand the relationship between the intervention components and HCV screening; and 3) reduce HCV-related morbidity and mortality.

86185

Food Cost and Perceptions: through the lens of coaches providing family-based childhood obesity treatment

Melissa Ramel, PhD, MPH, RD, LD, Denise Wilfley, PhD and Rachel Tabak, PhD, RD

Washington University in St. Louis School of Medicine

ABSTRACT IMPACT: This work will help to identify ways to adapt family-based obesity treatment based on families' food purchasing behaviors and beliefs. OBJECTIVES/GOALS: Families in obesity 